\title{
Duplication of linear algebras
}

\author{
By I. M. H. Etherington.
}

(Received and read 3rd May, 1940.)

The process of duplication of a linear algebra was defined in an earlier paper ${ }^{1}$, where its occurrence in the symbolism of genetics was pointed out. The definition will now be repeated with an amplification. Although for purpose of illustration it is applied to the algebra of complex numbers, duplication will seem of no special significance if attention is fixed on algebras with associative multiplication and unique division; for duplication generally destroys these properties. The results to be proved, however, show that it is significant in connection with various other conceptions which appeared in the discussion of genetic algebras; namely baric algebras and train algebras (defined in G.A.), also nilpotent algebras, linear transformation and direct multiplication of algebras.

\section{$\S 1$. Meaning of duplication.}

Let $X$ be a linear algebra of order $n$ over the field $F$, with basis $a^{1}, a^{2}, \ldots a^{n}$, having the multiplication table

$$
a^{\mu} a^{\nu}=\sum_{\sigma=1}^{n} \gamma_{\sigma}^{\mu y} a^{\sigma}, \quad(\mu, \nu=1, \ldots n), \quad\left(\gamma_{\sigma}^{\mu \nu}<F\right)
$$

The commutative and associative laws of multiplication are not assumed. We shall write

$$
X=\left(a^{1}, a^{2}, \ldots a^{n}\right) .
$$

Except for the positive integer $n$, italic letters will be used consistently for hypercomplex numbers, or as they will be called elements; and except in the enumerating indices (which always run from $\mathrm{I}$ to $n$ ) greek letters (other than $\Sigma$ ) will be used consistently for elements of $F$, which will be called numbers. Also $\Sigma$ will always denote summation with respect to repeated indices. Thus we may without ambiguity

1 Etherington, “Genetic algebras,” Proc. Roy. Soc., Edin., 59 (1939), 242-258. Reference will also be made to "On non-associative combinations," ibid., 153-162. These papers will be referred to as G.A. and N.C. Cf. also ibid. (B), 61 (1941), 24-42. 
define $X$ by writing instead of (1.2) and (1.1)

where

$$
X=\left(a^{\mu}\right) \text {, }
$$

We have then

$$
a^{\mu} a^{\nu}=\Sigma \gamma_{\sigma}^{\mu \nu} a^{\sigma} .
$$

Writing

$$
a^{\mu} a^{\nu} \cdot a^{\theta} a^{\phi}=\Sigma \gamma_{\sigma}^{\mu \nu} a^{\sigma} . \Sigma \gamma_{\tau}^{\theta \phi} a^{\tau}=\Sigma \gamma_{\sigma}^{\mu \nu} \gamma_{\tau}^{\theta \phi} a^{\sigma} a^{\tau} .
$$

this becomes

$$
a^{\mu} a^{\nu}=a^{\mu \nu}
$$

which may be regarded as the multiplication table of another linear algebra over the same field $F$, denoted

$$
X^{\prime}=\left(a^{\mu \nu}\right)
$$

and called the duplicate of $X$.

It was assumed in G. A. that $X$ was commutative; accordingly no distinction was drawn between $a^{\mu \nu}$ and $a^{\nu \mu}$, and $X^{\prime}$ was a commutative algebra of order $\frac{1}{2} n(n+1)$. It was also pointed out that when $X$ is non-commutative, the non-commutative algebra $X$ ' is of order $n^{2}$.

In the case when $X$ is commutative, however, it is still possible in carrying out the process $(1.5,6,7)$ to draw a formal distinction between $a^{\mu \nu}$ and $a^{\nu \mu}$, and thus to obtain a non-commutative duplicate algebra of order $n^{2}$ instead of $\frac{1}{2} n(n+1)$. Its multiplication table will still be (1.7), but these equations will now number $n^{4}$ instead of

$$
\frac{1}{2} \cdot \frac{1}{2} n(n+1) \cdot\left\{\frac{1}{2} n(n+1)+1\right\}=\frac{1}{8} n(n+1)\left(n^{2}+n+2\right) .
$$

(Provided that the order of the subalgebra $X^{2}$ is not less than 2 , multiplication will be non-commutative in the non-commutative duplicate algebra.)

Consider, for example, the algebra of complex numbers, $Z=(1, i)$ where $1^{2}=1,1 i=i l=i, i^{2}=-1$. Its commutative duplicate is $Z^{\prime}=\left(1^{2}, 1 i=i 1, i^{2}\right)$, and its non-commutative duplicate is $Z^{\prime}=\left(1^{2}\right.$, $\left.1 i, i 1, i^{2}\right)$, with multiplication tables respectively

$$
\begin{array}{rl|rrr} 
& a & b & c \\
1 i= & =a \\
i 1 & =b & a & b & -a \\
i^{2} & =c & & &
\end{array}
$$

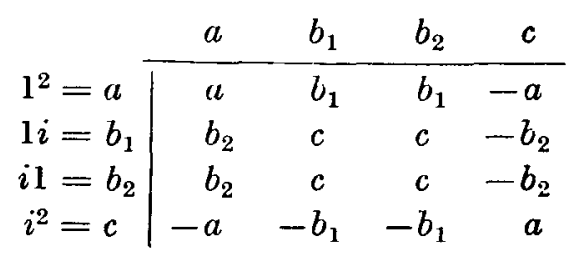

(1.11) 
These may be contrasted with the "direct square," or direct. product of two algebras isomorphic with $Z$, say

$$
Z Z_{1}=(1, i) \times(I, j)=(1 I, i I, 1 j, i j),
$$

having the commutative multiplication table

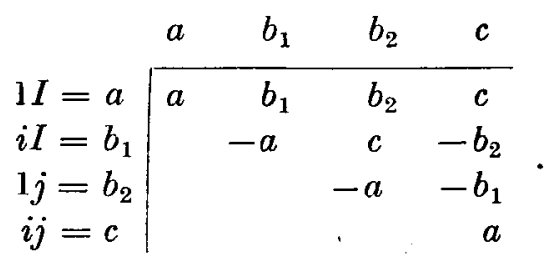

Like $Z$, this is commutative and associative, and possesses a 1-element (having the properties of 1 and $I$ in the factor algebras), namely $a$; and it has the property of unique division. On the other hand both duplicate algebras are non-associative; and it follows Theorem II (i), (ii), infra, that except in the trivial case $n=1$ a duplicate algebra cannot be a division algebra or possess a 1-element.

Returning to the general commutative algebra $X$, and supposing its order $>2$, we can if desired draw distinctions between $a^{\mu \nu}$ and $a^{p \mu}$ in some cases but not all (e.g. regard $a^{12}=a^{21}$, but other $a^{\mu \nu} \neq a^{\nu \mu}$ ), and thus obtain intermediate part-commutative duplicate algebras, of any order between $n^{2}$ and $\frac{1}{2} n(n+1)$.

In the rest of this paper, except where otherwise indicated, it is optional whether we assume that $X$ is non-commutative, in which case $X^{\prime}$ is unique; or that $X$ is commutative and that one of its duplicates is selected as the duplicate and denoted $X^{\prime}$. The meaning of the phrase quadratic form is fixed accordingly: a quadratic form in $X$ means a linear combination (coefficients in $F$ ) of those products of base elements which are distinguished as corresponding to the base elements of $X^{\prime}$.

\section{§2. General properties of a duplicate algebra.}

To any element

$$
x^{\prime}=\sum a_{\mu \nu} a^{\mu \nu}
$$

of $X^{\prime}$, there corresponds the quadratic form $\Sigma a_{\mu \nu} a^{\mu} a^{\nu}$ in $X$. The element $x$ ' and the quadratic form will be called isomorphs of each other. The correspondence is unique both ways, and under it the operations of addition and multiplication both hypercomplex and sealar are conserved. 
Also, in virtue of (1.6) and the multiplication table (1.4), to any element (2.1) of $X$, there corresponds a unique element of $X$, called the homomorph of $x$ '. Again, under this correspondence addition and both kinds of multiplication are conserved; but the correspondence is not unique in the opposite direction. It is nevertheless sometimes convenient (especially in the genetical symbolism) to use " =" for both correspondences, and thus to write:

$$
\begin{aligned}
\sum \alpha_{\mu \nu} a^{\mu \nu} & =\sum a_{\mu \nu} a^{\mu} a^{\nu} \quad \text { (its isomorph) } \\
& =\sum a_{\mu \nu} \gamma_{\sigma}^{\mu \nu} a^{\sigma} \text { (its homomorph). }
\end{aligned}
$$

Not all elements of $X$ are homomorphs: in order that $x$ should be a homomorph, it is necessary and sufficient that it should be a linear combination of the elements $\Sigma \gamma_{\sigma}^{\mu \nu} a^{\sigma}$, i.e. of $a^{\mu} a^{\nu}$; in other words it must belong to the invariant subalgebra $X^{2}$. Thus the homomorphism is a mapping of $X^{\prime}$ on $X^{2}$.

When forming a product in $X^{\prime}$, we may replace the elements to be multiplied by their homomorphs in $X$, and then multiply, leaving the product in quadratic form and taking its isomorph in $X^{\prime}$. In symbols, if

then

$$
x^{\prime}=\sum a_{\mu \nu} a^{\mu \nu}, \quad y^{\prime}=\sum \beta_{\theta \phi} a^{\theta \phi},
$$

$$
x^{\prime} y^{\prime}=\sum \alpha_{\mu \nu} \gamma_{\sigma}^{\mu \nu} a^{\sigma} . \Sigma \beta_{\theta \phi} \gamma_{\tau}^{\theta \phi} a^{\tau}=\Sigma a_{\mu \nu} \beta_{\theta \phi} \gamma_{\sigma}^{\mu \nu} \gamma_{\tau}^{\theta \phi} a^{\sigma \tau}
$$

which is evidently the correct result. We deduce immediately ${ }^{1}$ THEOREM I. In forming any product, power or continued product in $X^{\prime}$, we can perform all the operations on the homomorphs in $X$, only in the final multiplication leaving the product in quadratic form: its isomorph in $X^{\prime}$ will then be the result required. (The operations have to be. performed in a definite order since multiplication is non-associative.)

Elements of $X^{\prime}$ whose homomorphs are zero will be called $o$-elements; they form a linear set which will be denoted $O$.

Theorem II. (i) Assuming $n>1, X$ ' necessarily contains o-elements other than zero, so that $O \neq 0$. (ii) In $X^{\prime}$, any product which contains an o-element as one factor is zero. (iii) $O$ is an invariant subalgebra of $X^{\prime}$. (iv) The difference algebra $\left(X^{\prime}-O\right)$ is isomorphic with $X^{2}$.

1 In the genetical symbolism, this theorem corresponds to the fact that in order to obtain the distribution of zygotic types of an $r^{\text {th }}$ filial generation, provided that no selection acts on the zygotes, it is sufficient to trace only the gametic distribution through the $r-1$ intervening generations. 
For if $n>1$, the elements $\Sigma \gamma_{\sigma}^{\mu \nu} a^{\sigma}$ (i.e. $a^{\mu} a^{\nu}$ ) are more than $n$ in number, and therefore cannot be linearly independent; this is equivalent to the statement (i). (ii) follows from Theorem I, and (iii) is an immediate consequence. Or (iii) and (iv) together follow from the general properties of homomorphisms ${ }^{2}$.

In the algebras $Z^{\prime}$ of $\S 1$, write

$$
a+c=o, \quad b_{1}-b_{2}=o^{\prime}:
$$

these are o-elements. The multiplication tables $(1.10,11)$ become

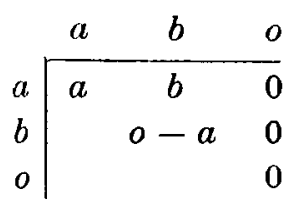

\begin{tabular}{ccccc} 
& $a$ & $b_{1}$ & $o$ & $o^{\prime}$ \\
\cline { 2 - 5 }$a$ & $a$ & $b_{1}$ & 0 & 0 \\
$b_{1}$ & $b_{1}-o^{\prime}$ & $o-a$ & 0 & 0 \\
$o$ & 0 & 0 & 0 & 0 \\
$o^{\prime}$ & 0 & 0 & 0 & 0
\end{tabular}

'The zeros in the tables illustrate Theorem II (ii), (iii); while the results of suppressing all the $o$ 's illustrate the isomorphism of $\left(Z^{\prime}-O\right)$ with $Z^{2}$, i.e. with $Z$.

By a polynomial in an element $x$, we shall mean a finite linear combination of powers of $x$, with coefficients in $F$. Since $X$ does not in general contain a l-element to serve as an interpretation of $x^{0}$ (and even if $X$ does, $X$ ' does not), we shall exclude from consideration polynomials with a constant term. Thus when multiplication is $(a)$ associative, $(b)$ commutative and non-associative, (c) non-commutative and non-associative, a polynomial means a finite expression (for the index notation see N.C., §2)

(a.) $\quad a x+\beta x^{2}+\gamma x^{3}+\delta x^{4}+\epsilon x^{5}+\ldots$.

(b) $\quad a x+\beta x^{2}+\gamma x^{3}+\delta x^{4}+\epsilon x^{2.2}+\zeta x^{5}+\ldots$.

(c) $\quad \alpha x+\beta x^{2}+\gamma x^{2+1}+\delta x^{1+2}+\epsilon x^{(2+1)+1}+\zeta x^{(1+2)+1}+\ldots$.

If $x$ is the homomorph of $x^{\prime}$, then a polynomial in $x^{\prime}$ has as homomorph the same polynomial in $x$ (perhaps compressed, if multiplication is associative or commutative in $X$ and not in $X^{\prime}$ ).

1 van der Waerden, Moderne Algebra (Berlin, 1930), I, pp. 56-57, where, since the postulate of associative multiplication in rings is not used, the results apply to nonassociative algebras. "Invariant subalgebra" is here called Ideal, and "difference algebra" Restklassenring. 
Suppose that every element $x=\Sigma \alpha_{\sigma} a^{\sigma}$ of $X$ satisfies the identity

$$
f\left(x, a_{\sigma}\right)=0 \text {, }
$$

where $f\left(x, a_{\sigma}\right)$ is a polynomial in $x$ whose coefficients are functions of the coordinates $a_{\sigma}$ of $x$. Then the function $f\left(x^{\prime}, \Sigma \alpha_{\mu \nu} \gamma_{\sigma}^{\mu \nu}\right)$, formed from any element $x^{\prime}$ of $X^{\prime}$ and the coordinates of its homomorph in the same way as $f\left(x, a_{\sigma}\right)$ is formed from $x$ and its coordinates, is an - element of $X^{\prime}$ whose homomorph is zero. Hence by Theorem II (ii),

Thus we have

$$
x^{\prime} . f\left(x^{\prime}, \Sigma \alpha_{\mu \nu} \gamma_{\sigma}^{\mu \nu}\right)=0, \quad f\left(x^{\prime}, \Sigma \alpha_{\mu \nu} \gamma_{\sigma}^{\mu \nu}\right) . x^{\prime}=0 .
$$

Theorem III. If every element $x=\Sigma a_{\sigma} a^{\sigma}$ of $X$ satisfies an identity (2.8), then every element $x^{\prime}=\Sigma a_{\mu \nu} a^{\mu_{\nu}}$ of $X^{\prime}$ satisfies the identities (2.9). If multiplication is associative or commutative in $X$ and not in $X^{\prime}$, the function $f$ in (2.9) may be interpretable in various ways.

For example, every element $z=a l+\beta i$ of $Z$ satisfies the rank equation

$$
z^{2}-2 \alpha z+a^{2}+\beta^{2}=0 .
$$

Theorem III can be applied not to this identity but to

$$
z^{3}-2 a z^{2}+\left(\alpha^{2}+\beta^{2}\right) z=0 .
$$

Using the notation (2.4), any element $z^{\prime}=a a+\beta b+\gamma o$ of the commutative duplicate algebra has the homomorph $a 1+\beta i$, and therefore satisfies

$$
z^{34}-2 \alpha z^{3}+\left(a^{2}+\beta^{2}\right) z^{\prime 2}=0,
$$

which is in fact the rank equation of $Z$ '. Similarly the element$z^{\prime}=\alpha a+\beta b+\gamma o+\delta o^{\prime}$ of the non-commutative duplicate satisfies the left and right rank equations

$$
\begin{aligned}
& z^{\prime 1+(1+2)}-2 a z^{1+2}+\left(\alpha^{2}+\beta^{2}\right) z^{2}=0 \\
& z^{\prime(2+1)+1}-2 \alpha z^{\prime 2+1}+\left(\alpha^{2}+\beta^{2}\right) z^{\prime 2}=0
\end{aligned}
$$

and also satisfies

$$
\begin{aligned}
& z^{\prime 1+(2+1)}-2 \alpha z^{1+2}+\left(\alpha^{2}+\beta^{2}\right) z^{\prime 2}=0 \\
& z^{\prime(1+2)+1}-2 \alpha z^{\prime 2+1}+\left(\alpha^{2}+\beta^{2}\right) z^{\prime 2}=0
\end{aligned}
$$

\section{§3. Related algebras duplicated.}

Theorem IV. If part-commutative duplicate algebras are excluded, the duplicate of a linear transform of $X$ is a linear transform of $X^{\prime}$.

For if the equations of transformation of $X$ are

$$
b^{a}=\Sigma \lambda_{\mu}^{a} a^{\mu}, \quad a^{\mu}=\Sigma \Lambda_{\alpha}^{\mu} b^{a}
$$

the multiplication table (1.4) becomes

$$
b^{a} b^{\beta}=\Sigma \lambda_{\mu}^{a} \lambda_{\nu}^{\beta} \gamma_{\sigma}^{\mu \nu} \Lambda_{\varepsilon}^{\sigma} b^{e}
$$


Duplicating (commutatively or non-commutatively), we obtain

$$
\begin{aligned}
b^{a \beta} b^{\gamma \delta} & =\Sigma\left(\lambda_{\mu}^{a} \lambda_{\nu}^{\beta} \gamma_{\sigma}^{\mu \nu} \Lambda_{\epsilon}^{\sigma}\right)\left(\lambda_{\theta}^{\gamma} \lambda_{\phi}^{\delta} \gamma_{\tau}^{\theta \phi} \Lambda_{\xi}^{\tau}\right) b^{\epsilon \xi} \\
& =\Sigma\left(\lambda_{\mu}^{a} \lambda_{\nu}^{\beta}\right)\left(\lambda_{\theta}^{\gamma} \lambda_{\phi}^{\delta}\right) \gamma_{\sigma}^{\mu \nu} \gamma_{\tau}^{\theta \phi}\left(\Lambda_{\epsilon}^{\sigma} \Lambda_{\xi}^{\tau} b^{\epsilon \xi}\right)
\end{aligned}
$$

and this is precisely the result which would be obtained by applying to the duplicate multiplication table (1.7) (commutative or noncommutative correspondingly) the transformation

$$
b^{a \beta}=\Sigma \lambda_{\mu}^{a} \lambda_{\nu}^{\beta} a^{\mu \nu}, \quad a^{\mu \nu}=\Sigma \Lambda_{a}^{\mu} \Lambda_{\beta}^{\nu} b^{a \beta} .
$$

If $X^{\prime}$ is $(a)$ a commutative duplicate algebra, or (b) a noncommutative duplicate algebra, it will be seen ${ }^{1}$ that the matrix of the induced transformation (3.4) in either direction is (a) the Schläflian (or second induced matrix), or (b) the direct square (or second Burnside matrix), of the matrix of the original transformation (3.1).

Similarly it is easy to prove

THEOREM V. The commutative or non-commutative duplicate of the direct product of two algebras coincides with the direct product of their commutative or non-commutative duplicates. Conversely, the direct product of any two duplicate algebras coincides with a duplicate of their direct product.

\section{$\S 4$. Algebras of special type duplicated.}

(a) Nilpotent algebras².

Suppose that $X$ is nilpotent of degree $^{3} 2 \delta$; i.e. in $X$ all products of $2 \delta$ factors vanish. It will be shown that $X^{\prime}$ is nilpotent of degree $2 \delta-1$.

${ }^{1}$ See, e.g., Aitken, Proc. London Math. Soc. (2), 38 (1935), 354-376.

2 In this section, as in N.C. $\S 3, \delta, a$ denote positive integers.

${ }^{3}$ Index is the usual word in this context : $c f$. Wedderburn, Proc. London Math. Soc. (2), 6 (1908), 77-118; p. 111. But having drawn a distinction in N.C. between index and degree, I find the latter word more appropriate here. It is perhaps not irrelevant to point out an error in Wedderburn's paper, concerning nilpotent non. sssociative algebras. It is stated (loc. cit., p. 111) that the sum of all the $r^{\text {th }}$ powers of such an algebra is less than (i.e. is contained in but is not equal to) the sum of the $(r-1)^{\text {th }}$ powers. This is not true of the commutative algebra $X=(a, b, c)$ where $a^{2}=b$, $a b=b^{2}=c, a c=b c=c^{2}=0$; for which $X^{2}=(b, c), X^{3}=(c), X^{4}=0, X^{2.2}=(c)$, $X^{5}=X^{2.2+1}=X^{3+2}=0$. For $X$ is nilpotent of degree 5 , whereas $X^{4}+X^{0.2}=X^{3}$. Cf. Etherington, "Special train algebras," Quart. Journ. Math. (in press). 
Consider first a product $a^{\prime}$ in $X^{\prime}$ containing $\delta$ factors. It is a linear combination of products of $\delta$ base elements. Each product of $\delta$ base elements is isomorphic with a product of $2 \delta$ base elements in $X$, and is therefore an $o$-element. Now consider a product $x^{\prime}$ of $2 \delta-1$ factors in $X^{\prime}$ : it is expressible as $a^{\prime} b^{\prime}$, where either $a^{\prime}$ or $b^{\prime}$ contains at least $\delta$ factors; i.e. either $a^{\prime}$ or $b^{\prime}$ is an o-element, and hence (Theorem II) $x^{\prime}=0$; as was to be proved. The same argument applies a fortiori if $X$ is nilpotent of degree $2 \delta-1$.

Or suppose ( $c f$. N.C., p. 156) that $X$ is nilpotent of altitude $a$; i.e. in $X$ all products of altitude $a$ vanish. A product $a^{\prime}$ in $X^{\prime}$ of altitude $a-1$ is a linear combination of products of base elements having the same altitude, each isomorphic with a product of altitude $a$ in the base elements of $X$; and is therefore an o-element. A product $x^{\prime}$ of altitude $a$ is expressible as $a^{\prime} b^{\prime}$, where either $a^{\prime}$ or $b^{\prime}$ is of altitude $a-1$ and is thus an $o$-element; so that $x^{\prime}=0$.

We have thus proved:

Theorem VI. If $X$ is nilpotent (i) of degree $2 \delta-1$ or $2 \delta$, or (ii) of altitude a, then $X^{\prime}$ is nilpotent (i) of degree $28-1$, or (ii) of altitude $a$, accordingly.

(b) Baric and train algebras.

If $X$ is a baric algebra (G.A., $\S 3$ ), there exists for any element $x$ a number $\xi(x)$, the weight of $x$, such that

$$
\dot{\xi}(x+y)=\xi(x)+\xi(y), \xi(x y)=\xi(x) \xi(y), \xi(\alpha x)=\alpha \xi(x) .
$$

If $x^{\prime}$ is any element of $X^{\prime}$, with homomorph $x$, and we define $\xi\left(x^{\prime}\right)$ as being equal to $\xi(x)$, then it follows that

$$
\xi\left(x^{\prime}+y^{\prime}\right)=\xi\left(x^{\prime}\right)+\xi\left(y^{\prime}\right), \xi\left(x^{\prime} y^{\prime}\right)=\xi\left(x^{\prime}\right) \xi\left(y^{\prime}\right), \xi\left(a x^{\prime}\right)=a \xi\left(x^{\prime}\right) ;
$$

so that $\xi\left(x^{\prime}\right)$ is a weight function of $X^{\prime}$. Moreover, if

then

$$
\xi\left(a^{\mu}\right)=\xi^{\mu},
$$

Thus we have

$$
\xi\left(a^{\mu \nu}\right)=\xi\left(a^{\mu} a^{\nu}\right)=\xi^{\mu} \xi^{\nu}
$$

Theorem VII. If $X$ is a baric algebra with weight vector $\xi^{\mu}$, then $X^{\prime}$ is a baric algebra with weight vector $\xi^{\mu} \xi^{\nu}$, and the weight of any element in $X^{\prime}$ is equal to the weight of its homomorph in $X$.

Combining this with Theorem III, we obtain 
TheOREm VIII. If $X$ is a train algebra with (left or right) principal train roots $1, \lambda, \mu, \ldots$, then $X^{\prime}$ is a train algebra with (left or right) principal train roots included in $1,0, \lambda, \mu, \ldots$ Instances of this theorem were observed in G.A.

It may be stated that the duplicate of a special train algebra (G.A., p. 246), although a train algebra, is not always a special train algebra ${ }^{1}$. The question, which was left open in G.A., whether a train algebra is necessarily a special train algebra, is thus to be answered in the negative.

1 The statement (G.A., p. 247) that "all the fundamental genetic algebras are special train algebras" refers to gametic algebras, not to the zygotic algebras which are derived from them by duplication.

Mathematical Institute,

16 Chambers Street, EdinRURGH. 